\title{
Safety and efficacy of nimotuzumab combined with chemoradiotherapy in Chinese patients with locally advanced cervical cancer
}

This article was published in the following Dove Press journal:

OncoTargets and Therapy

17 August 2017

Number of times this article has been viewed

\section{Yong-Fa Chen \\ Wu-Bin Tang \\ Xin-Xi Pan \\ Chu-Rong Wu \\ Yang Cao \\ Wen Yang}

Department of Oncology, People's Hospital of Nanhai District, Southern Medical University, Foshan, People's Republic of China
Correspondence: Wen Yang Department of Oncology, People's Hospital of Nanhai District, Southern Medical University, Foshan-528200, People's Republic of China Tel/fax +86 75786399206 Email yangwen 149@hotmail.com
Objective: To evaluate efficacy and safety of nimotuzumab combined with chemotherapy and radiotherapy in women with locally advanced cervical cancer.

Materials and methods: Women with locally advanced cervical cancer (stage IIB, III, or IVA) who experienced relapse after first-line chemoradiotherapy and one or more lines of palliative chemotherapy were enrolled. All patients received nimotuzumab weekly at $200 \mathrm{mg} / \mathrm{m}^{2}$ as single agent for 4 weeks (induction phase), then concurrent with 6 cycles (21-day per cycle) of gemcitabine $\left(800 \mathrm{mg} / \mathrm{m}^{2}\right)$ or cisplatin $\left(50 \mathrm{mg} / \mathrm{m}^{2}\right)$ for 18 weeks (concurrent phase) and then once every 2 weeks (maintenance phase). Overall response rate (ORR) was assessed after 4 weeks of induction therapy and then every 3 months according to response evaluation criteria in solid tumors version 1.1 (primary end point). Secondary end points include progression-free survival (PFS), overall survival (OS), and drug toxicity. Descriptive statistics was used for ORR, and Kaplan-Meier curves were generated for OS and PFS.

Results: A total of 80 women with locally advanced cervical cancer were enrolled and evaluated for safety and efficacy. Our results demonstrated that none of the patients had a complete response $(0 \%), 11$ patients had a partial response (14\%), and 10 patients had progressive disease $(13 \%)$, giving a tumor response rate of $14 \%$. A total of 59 patients had stable disease (74\%), giving a disease control rate of $88 \%$ (70/80). Median PFS was 8.21 months (95\% confidence interval [CI]: 5.09-12.45). Median OS was 11.96 months (95\% CI: 8.11-23.95). The most common adverse events were mucositis, myelosuppression, and gastrointestinal disturbance.

Conclusion: Our study results suggested that nimotuzumab in combination with chemotherapy and radiotherapy is well tolerated, and could be a better treatment alternative in patients with locally advanced cervical cancer.

Keywords: nimotuzumab, metastatic cervical cancer, Chinese patients, radiotherapy, chemoradiotherapy

\section{Introduction}

Cervical cancer is the second most common type of cancer for women worldwide, and it is also one of the most preventable types of cancer. It is fourth most commonly diagnosed cancer worldwide, and the fourth leading cause of cancer death in women, accounting for $9 \%$ of total new cancer cases and $7.5 \%$ of total cancer deaths in females. ${ }^{1,2}$ Cervical cancer is very common in developing countries ( $15 \%$ of cases), whereas in developed countries cervical cancer accounts for only $3.6 \%{ }^{3}$

Most women who are found to have cervical cancer were not screened regularly. ${ }^{4,5}$ As a result, about $25 \%$ of patients with cervical cancer present with locally advanced disease (stage IIB through IVA according to the staging system of the International 
Federation of Gynecology and Obstetrics). ${ }^{5-7}$ Among the patients with locally advanced cervical cancer, 1 out of 3 patients have treatment failure even with the most effective platinum-based doublet chemotherapy with concurrent radiation. ${ }^{8}$ Only a small proportion of patients with recurrent disease can be cured by either surgery or radiation; however, most are not, hence, systemic palliative chemotherapy is considered as the only option for patients with recurrent locally advanced cervical cancer. ${ }^{9}$

Currently, platinum-based chemotherapy (cisplatin) with taxanes (topotecan and paclitaxel) and gemcitabine is considered standard therapy because it produces similar response rates, progression-free survival (PFS), overall survival (OS) rates, and quality-of-life outcomes..$^{10} \mathrm{~A}$ study evaluated the efficacy of bevacizumab in combination with chemotherapy in advanced cervical cancer; the results of this study showed that the addition of bevacizumab to chemotherapy regimen significantly improve PFS and OS. ${ }^{11}$ However, these results were not consistent with phase II clinical study in which bevacizumab was combined with the cisplatin-topotecan therapy in patients with recurrent or persistent cervical cancer. ${ }^{11}$

Overexpression of epidermal growth factor receptor (EGFR) in cervical cancer is well documented (overexpression ranges from $6 \%$ to $90 \%$ ); this makes EGFR as a potential target in cervical cancer. ${ }^{12}$ Nimotuzumab is a humanized IgG1 monoclonal antibody which blocks the activation of EGFR without intrinsic stimulating activity. Nimotuzumab displayed a longer plasma half-life with better safety profile when compared with other anti-EGFR antibodies. ${ }^{13,14}$ Nimotuzumab is approved in some countries for the treatment of glioma and advanced head and neck cancer as a single agent or in combination with chemotherapy and radiotherapy..$^{12}$ Efficacy and safety of nimotuzumab as a single agent or in combination with chemotherapy and radiation have been evaluated in cervical cancer; however, no results have been published. A pilot study was conducted to evaluate efficacy and safety of nimotuzumab in combination with chemotherapy among Caucasian patients with advanced or progressive cervical cancer. ${ }^{12}$ The results of this study showed that nimotuzumab is well tolerated and may have a role in the treatment of advanced cervical cancer. However, to date, there has been no study that evaluates the efficacy and safety of nimotuzumab in combination with chemoradiotherapy in Chinese patients with locally advanced cervical cancer. We, therefore, designed pilot, phase IIa, prospective, single-arm study to evaluate efficacy and safety of nimotuzumab in combination with chemoradiotherapy in Chinese patients with locally advanced cervical cancer. Our study will serve as the basis for conducting large multi-centric randomized clinical study among Chinese patients with locally advanced cervical cancer.

\section{Methods}

\section{Patients}

In this pilot, phase IIa, prospective, open-label, singlearm study, women with locally advanced cervical cancer who experienced disease relapse after receiving first-line chemoradiotherapy and one or more lines of palliative chemotherapy were enrolled in the study from January 2010 to January 2015. The patients were enrolled at Department of Oncology, People's Hospital of Nanhai District, Southern Medical University, People's Republic of China. All patients provided written informed consent before screening and other study-related procedures, and the study was approved by the ethics committee of the Southern Medical University.

Women with histologically and/or radiologic confirmed diagnosis of squamous-cell carcinoma, adenosquamous carcinoma or adenocarcinoma of the cervix of International Federation of Gynecology and Obstetrics stage IIB (localized disease with parametrial involvement), stage III (extension of the tumor to the pelvic wall) or stage IVA (involvement of the bladder or rectal mucosa) were enrolled. All patients had measurable disease and were required to have an Eastern Cooperative Oncology Group (ECOG) score of 0-2, and at least 1 prior systemic chemotherapy regimen for metastatic recurrent disease administered at least 3 weeks before starting protocol therapy. Other eligibility criteria were as follows: absolute neutrophil count $\geq 1.5 \times 10^{9} / \mathrm{L}$, platelets count $\geq 100 \times 10^{9} / \mathrm{L}$, total bilirubin, aspartate aminotransferase (AST) and alanine aminotransferase (ALT) $\leq 1.5 \times$ upper limit of normal and calculated creatinine clearance (Cockroft) $\geq 50 \mathrm{~mL} / \mathrm{min}$. The patients with uncontrolled systemic disease or infection, pregnancy or breastfeeding, and previous or concurrent malignancy other than adequately treated nonmelanoma skin cancer were excluded.

\section{Treatment}

\section{Antibody and chemotherapy}

All the enrolled patients received intravenous infusion of nimotuzumab (monoclonal antibody) alone at a dose of $200 \mathrm{mg} / \mathrm{m}^{2}$ for 30 minutes weekly during the first 4 weeks (induction phase) followed by 18 weekly applications of nimotuzumab concurrently with 6-21 days cycles of singleagent chemotherapy (concurrent). After week 18 (22 weeks since treatment initiation), patients continued with nimotuzumab alone (maintenance) once every 2 weeks at the same 
dose until unacceptable toxicity or consent withdrawal. No premedication including rash prophylaxis was administered before nimotuzumab infusion. Single-agent chemotherapy comprised intravenous infusion of gemcitabine $\left(800 \mathrm{mg} / \mathrm{m}^{2}\right)$ for 30 minutes, or cisplatin $\left(50 \mathrm{mg} / \mathrm{m}^{2}\right)$ was administered intravenously in an outpatient setting.

\section{Radiotherapy}

All the enrolled patients received radiotherapy. Radiotherapy was administered to the whole pelvic region in 24 fractions totaling $40.8 \mathrm{~Gy}$ or 30 fractions totaling $51.0 \mathrm{~Gy}$. The total dose delivered was 40 Gy in patients with stage IIB, and 30 Gy in patients with stage III or IVA disease. The total dose delivered to point $\mathrm{A}$ (a reference location $2 \mathrm{~cm}$ lateral and $2 \mathrm{~cm}$ superior to the cervical OS) was $80.8 \mathrm{~Gy}$ in patients with stage IIB and 81.0 Gy in patients with stage III or IVA disease; the total dose delivered to point B (the pelvic wall) was 55.0 Gy in patients with stage IIB disease and 60.0 Gy in patients with stage III or IVA disease. Pelvic radiation was delivered by a four-field box technique (anteroposterior, posteroanterior and two lateral fields) with an X-ray energy of at least 4- $\mathrm{mV}$ photons. The pelvic field extended from the upper margin of L5 to the midportion of the obturator foramen or the lowest level of disease, with a $3-\mathrm{cm}$ margin, and laterally $1.5-2 \mathrm{~cm}$ beyond the lateral margins of the bony pelvic wall (at least $7 \mathrm{~cm}$ from the midline). The duration of the radiotherapy was 10 weeks. Radiotherapy was withheld if a patient had a leukocyte count of $<2,000 / \mathrm{mm}^{3}$, and delays of up to 1 week were also allowed in the event of radiationrelated gastrointestinal or genitourinary toxicity. ${ }^{5}$

\section{Efficacy and safety assessment}

Each patient was required to undergo a complete physical examination, a pelvic examination under anesthesia, chest radiography and intravenous pyelography or abdominal computed tomography to determine the clinical stage of the cancer. Baseline examination included a complete history and physical examination, complete blood cell count, blood chemistry and imaging by computed tomography (CT) or magnetic resonance imaging (MRI) of target lesions (4 weeks before starting the study). Primary end point was overall response rate. Overall response rate was defined as the proportion of enrolled patients who qualify for the analysis population of interest and who have a best response of partial response (PR) or complete response (CR) according to the response evaluation criteria in solid tumors (RECIST) 1.1 guidelines. Overall response rate was evaluated from patient's inclusion after 4 weeks of induction therapy and then every 3 months until second year of follow-up for each patient. Treatment response was evaluated according to the RECIST criteria (1.1) by the same imaging modality used for baseline evaluation. Disease control rate was defined as the proportion of enrolled patients, qualified for the analysis population of interest, having a best response of $\mathrm{PR}, \mathrm{CR}$ or stable disease (SD) according to RECIST 1.1 guidelines. Secondary end points include PFS, OS, and drug toxicity. PFS was defined as the time elapsed since the beginning of interventions until progressive disease is documented by growth of measurable lesions or new lesions on CT scan or MRI according to the response evaluation criteria in solid tumors (time frame: after patient's inclusion every 3 months for a period 2 years). OS was defined as the time from date of enrollment until date of death due to any cause (time frame: from patient's inclusion until 24 months). Drug toxicity was assessed with hematologic, renal, and hepatic laboratory measures as well as patient symptomatology and physical findings. Any abnormality in these parameters was reported as an adverse event according to the Common Terminology Criteria for Adverse Events version 3.0 (CTCAE v3.0). Adverse events were recorded according to CTCAE v3.0 at each visit before treatment administration. Follow-up assessments were made 4 weeks after the last dose of study medication and every 3 months thereafter.

\section{Statistical analysis}

A sample size of 75 evaluable patients with histologically confirmed diagnosis of locally advanced cervical cancer was sufficient to detect an assumed clinically significant difference of $11 \%$ in response rate after treatment with nimotuzumab combined with radiotherapy and chemotherapy, using a two-tailed test with $80 \%$ power and $5 \%$ level of significance. Considering dropout rate of $5 \%$, adjusted sample size was $\sim 80$ patients of histologically confirmed diagnosis of locally advanced cervical cancer. Hence, a total of 80 patients with histologically confirmed diagnosis of locally advanced cervical cancer required to enroll in this study to complete 75 evaluable patients, to evaluate efficacy and safety of nimotuzumab in combination with chemoradiotherapy in Chinese patients with locally advanced cervical cancer. Patients who had a presence of measurable disease and had at least 1 baseline and 1 postbaseline tumor measurement were considered to be tumor response-qualified population. Overall response rate and disease control rate end points were presented using descriptive statistics for tumor responsequalified patients. Kaplan-Meier curves were generated for OS and PFS for enrolled population (all patients who 
met eligibility criteria and enrolled). Median survival time together with $95 \%$ confidence interval (CI) was calculated. Data were analyzed using Graph Pad Prism statistical analysis software (version 6.0).

\section{Results}

\section{Patient characteristics}

Between January 2010 and January 2015, a total of 80 women with locally advanced cervical cancer were enrolled and treated with nimotuzumab combined with chemotherapy and radiotherapy. Data from all 80 patients were subjected to statistical analysis. The key clinicopathological characteristics are shown in Table 1 . The median age was 59 years (range 24-78), most were squamous cell carcinomas and had an ECOG performance status of 1 . The majority of patients had stage III and stage IVA of cervical cancer.

\section{Efficacy}

Of total 80 patients with locally advanced cervical cancer, no patient had a CR $(0 \%), 11$ patients had a PR (14\%), and 10 patients had progressive disease (13\%), giving an overall response rate of $14 \%$ (Table 2). A total of 59 patients had SD (74\%), giving a disease control rate of $88 \%$ (70/80). Median PFS was 8.21 months (95\% CI: 5.09-12.45). Median OS was 11.96 months (95\% CI: 8.11-23.95). The Kaplan-Meier curve of PFS (enrolled population) and OS (enrolled population) is presented in Figures 1 and 2, respectively.

\section{Safety}

The types and frequencies of treatment-related adverse events experienced in women with locally advanced cervical cancer after nimotuzumab plus chemoradiotherapy are

Table I Patients' characteristics

\begin{tabular}{lc}
\hline Variables & $\begin{array}{l}\text { Number of patients, } \\
\mathbf{N}=\mathbf{8 0}(\%)\end{array}$ \\
\hline $\begin{array}{l}\text { Age (years) } \\
\text { Median (minimum-maximum) }\end{array}$ & $59(24-78)$ \\
Pathological diagnosis & $48(60)$ \\
Squamous cell carcinoma & $22(28)$ \\
Adenocarcinoma & $10(12)$ \\
Adenosquamous & $65(8 I)$ \\
Eastern Cooperative Oncology Group performce status at baseline \\
I & I5 (19) \\
2 & \\
Staging of cervical cancer based on Federation of Gynecology and \\
Obstetrics \\
IIB & $38(48)$ \\
III & $25(3 I)$ \\
IVA
\end{tabular}

Table 2 Summary of tumor response (tumor-response qualified population)

\begin{tabular}{ll}
\hline Variables & $\begin{array}{l}\text { Number of patients, } \\
\mathbf{N}=\mathbf{8 0}(\%)\end{array}$ \\
\hline Complete response (CR) & 0 \\
Partial response (PR) & II (I4) \\
Tumor response rate (CR + PR) & $11(14)$ \\
Stable disease (SD) & $59(74)$ \\
Disease control rate (CR + PR + SD) & $70(88)$ \\
Progressive disease (PD) & $10(13)$ \\
\hline
\end{tabular}

shown in Table 3. The most common adverse events after nimotuzumab plus chemoradiotherapy were mucositis, myelosuppression, and gastrointestinal disturbance; however, no grade 4 events were registered. The grade 3 toxicity events observed were mucositis (10\%), gastrointestinal disturbance (3\%), leukocytopenia (4\%), anemia (4\%), thrombocytopenia $(5 \%)$, and radiodermatitis $(1 \%)$. The most frequent adverse events of all grades reported (occurs in $\geq 20 \%$ of patients) after nimotuzumab plus chemoradiotherapy were mucositis (45 [56\%] cases), febrile neutropenia (2 [5.4\%] cases), gastrointestinal disturbance (28 [35\%] cases), leukocytopenia (37 [46\%] cases), anemia (27 [34\%] cases), thrombocytopenia (35 [44\%] cases), granulocytopenia (22 [28\%] cases), and febrile neutropenia (20 [25\%] cases). Other less common adverse events of all grades reported after nimotuzumab plus chemoradiotherapy were (occurs in $\geq 15 \%$ of patients) nausea/vomiting (19\%), radiodermatitis (19\%), weight loss (16\%), anorexia (16\%), rash (16\%), and fatigue (15\%). There were no treatment-related deaths. No patient was discontinued from study and study drug due to adverse events. Overall, the safety data suggest that nimotuzumab combined with radiotherapy and chemotherapy has an acceptable safety profile in Chinese women with locally advanced cervical cancer.

\section{Discussion}

We present the first evidence that nimotuzumab combined with concurrent chemoradiotherapy in the treatment locally advanced cervical cancer. The results of this study demonstrate that nimotuzumab in combination with chemoradiotherapy is well tolerated and yields overall response rate of $14 \%$, disease control rate of $88 \%$ and disease stabilization in $74 \%$ of the patients. We have not observed CR in any of enrolled patients, this possibly due to the women of advanced cervical cancer were only enrolled. The observed effect translates into PFS $(95 \% \mathrm{CI})$ and OS $(95 \% \mathrm{CI})$ rates of 8.21 months (5.09-12.45) and 11.96 months (8.11-23.95), respectively. 


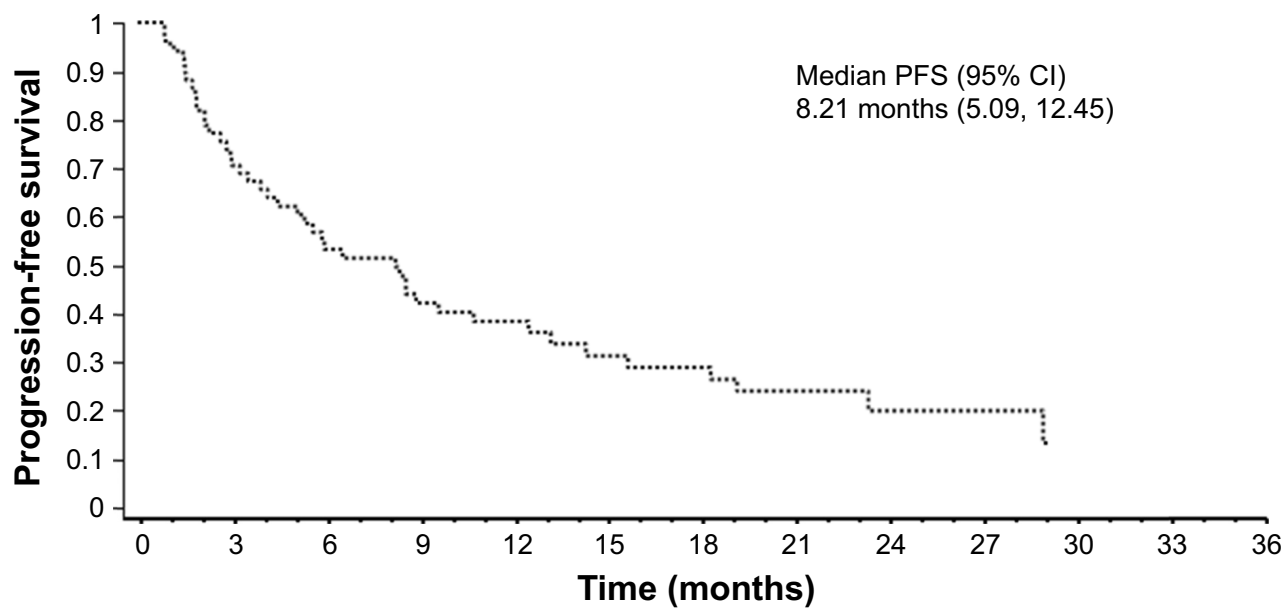

Figure I Kaplan-Meier curve of PFS (enrolled population).

Abbreviations: $\mathrm{Cl}$, confidence interval; PFS, progression-free survival.

EGFR is one of the key therapeutic targets for cancer treatment as it plays an important role in tumorigenesis and maintenance of cancers. Overexpression of EGFR is well documented in various types of malignancies including head and neck, colon, breast cancers and cervical cancer. ${ }^{15}$ In this setting, several EGFR inhibitors have been tested, including sunitinib, imatinib and temsirolimus but no evidence of any clinical benefit was observed. ${ }^{13,14}$ Among other monoclonal antibodies against the EGFR, only cetuximab has been evaluated in cervical cancer. The effect of cetuximab was tested in 5 patients with cervical cancer, only 1 patient reported with SD. ${ }^{16}$ In another clinical study, no clinical benefit was observed after treatment with cetuximab. ${ }^{17}$ Among EGFR inhibitors, nimotuzumab has demonstrated efficacy characterized by the induction of a long-term SD with a very low toxicity in patients with head and neck squamous cell carcinoma, pancreatic cancer, lung cancer and brain tumors. ${ }^{12,15}$ Nimotuzumab has already been approved for the treatment of advanced head and neck, glioma and esophageal cancer patients in combination with irradiation or chemoradiotherapy. ${ }^{12,15}$

The results of phase II study have suggested that nimotuzumab in combination with radiotherapy is well tolerated in locally advanced rectal cancer. ${ }^{18}$ Another pilot trial aimed to evaluate the efficacy and safety of nimotuzumab in Caucasian patients with advanced cervical cancer concluded that nimotuzumab is well tolerated and may have a role in the treatment of advanced cervical cancer. ${ }^{12}$ Our study results are in line with the previous study, ${ }^{12}$ however, the diseases' stabilization rate was better (74\%) when compared to Caucasian patients $(35 \%)$ who received nimotuzumab in combination with chemotherapy only. ${ }^{12}$ Moreover, in our study, the overall response rate and disease control rate after treatment of nimotuzumab in combination with chemotherapy and

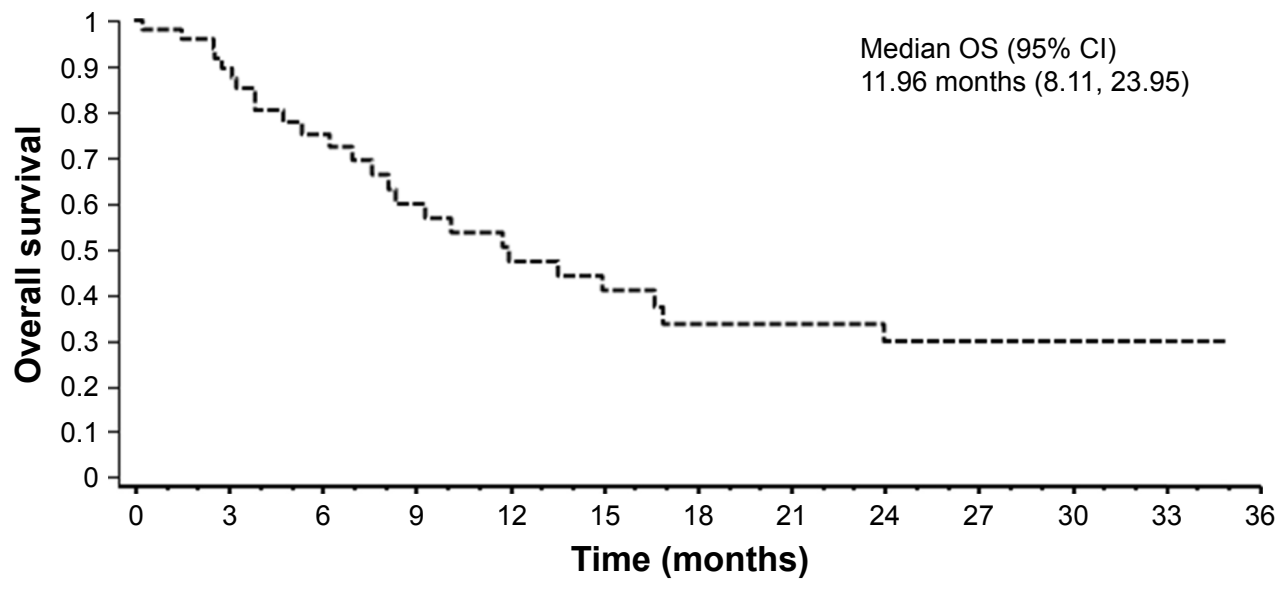

Figure 2 Kaplan-Meier curve of OS (enrolled population). Abbreviations: $\mathrm{Cl}$, confidence interval; OS, overall survival. 
Table 3 Summary of treatment-related adverse events experienced in women with locally advanced cervical cancer after nimotuzumab plus chemoradiotherapy

\begin{tabular}{llllll}
\hline Adverse events & Grade I, n (\%) & Grade 2, n (\%) & Grade 3, n (\%) & Grade 4, n (\%) & All grades, n (\%) \\
\hline Mucositis & $20(25)$ & $17(21)$ & $8(10)$ & $0(0)$ & $45(56)$ \\
Gastrointestinal disturbance & $18(23)$ & $8(10)$ & $2(3)$ & $0(0)$ & $28(35)$ \\
Leukocytopenia & $19(24)$ & $15(19)$ & $3(4)$ & $0(0)$ & $37(46)$ \\
Anemia & $17(21)$ & $7(9)$ & $3(4)$ & $4(0)$ & $27(34)$ \\
Thrombocytopenia & $18(23)$ & $13(16)$ & $0(0)$ & $0(0)$ & $35(44)$ \\
Granulocytopenia & $14(18)$ & $8(10)$ & $0(0)$ & $0(0)$ & $22(28)$ \\
Febrile neutropenia & $11(14)$ & $9(11)$ & $0(0)$ & $0(0)$ & $20(25)$ \\
Fatigue & $8(10)$ & $4(5)$ & $10)$ & $0(0)$ & $12(15)$ \\
Nausea/vomiting & $7(9)$ & $8(10)$ & $0(0)$ & $0(0)$ & $15(19)$ \\
Radiodermatitis & $8(10)$ & $6(8)$ & $0(0)$ & $0(0)$ & $15(19)$ \\
Rash & $7(9)$ & $6(8)$ & $0(0)$ & $0(0)$ & $13(16)$ \\
Anorexia & $8(10)$ & $5(6)$ & $0(0)$ & $0(0)$ & $13(16)$ \\
Proctitis & $6(8)$ & $0(5)$ & $0(0)$ & $0(0)$ & $10(13)$ \\
Fever & $7(9)$ & $2(3)$ & $0(0)$ & $0(0)$ & $19(9)$ \\
Pain & $9(1)$ & $5(6)$ & $0(0)$ & $13(16)$ \\
Weight loss & $8(10)$ & &
\end{tabular}

Note: Grade I indicates minimal effect, grade 2 indicates mild effect, grade 3 indicates moderate effect, and grade 4 indicates severe effect.

radiotherapy was $14 \%$ and $88 \%$, respectively. This indicates that the combination of nimotuzumab with chemotherapy and radiotherapy could be a better treatment option in patients with locally advanced cervical cancer.

\section{Conclusion}

Our study results suggested that nimotuzumab in combination with chemotherapy and radiotherapy is well tolerated, and could be a better treatment alternative in patients with locally advanced cervical cancer. Nimotuzumab combined with concurrent chemoradiotherapy has potential role in the treatment of locally advanced cervical cancer.

\section{Acknowledgments}

This work was supported by Foshan Medical Science and Technology Projects (No 2014AB000302). The authors thank all patients for their participation in the study. We would also like to thank Rakesh Ojha (senior consultant medical writer) for writing and editorial support in the preparation of this manuscript.

\section{Disclosure}

The authors report no conflicts of interest in this work.

\section{References}

1. Globocan. Estimated cancer incidence, mortality and prevalence worldwide in 2012. International Agency for Research on Cancer. Available from: http://globocan.iarc.fr/Pages/fact_sheets_cancer.aspx. Accessed April 3, 2017.
2. Cervical Cancer Overview. Available from: http://www.nccc-online. org/hpvcervical-cancer/cervical-cancer-overview/. Accessed April 4, 2017.

3. Mehmetoglu HC, Sadikoglu G, Ozcakir A, Bilgel N. Pap smear screening in the primary health care setting: a study from Turkey. $N \mathrm{Am} \mathrm{J}$ Med Sci. 2010;2(10):467-472.

4. Janerich DT, Hadjimichael O, Schwartz PE, et al. The screening histories of women with invasive cervical cancer, Connecticut. Am J Public Health. 1995;85(6):791-794.

5. Rose PG, Bundy BN, Watkins EB, et al. Concurrent cisplatin-based radiotherapy and chemotherapy for locally advanced cervical cancer. N Engl J Med. 1999;340(15):1144-1153.

6. SEER cancer incidence public use data base, 1973-1994. Bethesda, MD: National Cancer Institute; 1997 (software).

7. Cervical cancer. NIH consensus statement. NIH Office of Medical Applications of Research, Bethesda, MD, USA; 1996;14(1): $15-16$.

8. Duenas-Gonzalez A, Zarba JJ, Patel F, et al. Phase III, open-label, randomized study comparing concurrent gemcitabine plus cisplatin and radiation followed by adjuvant gemcitabine and cisplatin versus concurrent cisplatin and radiation in patients with stage IIB to IVA carcinoma of the cervix. J Clin Oncol. 2011;29(13):1678-1685.

9. Hockel M, Horn LC, Einenkel J. Laterally extended endopelvic resection: surgical treatment of locally advanced and recurrent cancer of the uterine cervix and vagina based on ontogenetic anatomy. Gynecol Oncol. 2012;127(2):297-302.

10. Monk BJ, Sill MW, McMeekin DS, et al. Phase III trial of four cisplatincontaining doublet combinations in stage IVB, recurrent, or persistent cervical carcinoma: a Gynecologic Oncology Group study. JClin Oncol. 2009;27:4649-4655.

11. Tewari KS, Sill MW, Long HJ 3rd, et al. Improved survival with bevacizumab in advanced cervical cancer. N Engl J Med. 2014;370(8): 734-743.

12. Cetina L, Crombet T, Jiménez-Lima R, et al. A pilot study of nimotuzumab plus single agent chemotherapy as second- or third-line treatment or more in patients with recurrent, persistent or metastatic cervical cancer. Cancer Biol Ther. 2015;16(5):684-689.

13. Zighelboim I, Wright JD, Gao F, et al. Multicenter phase II trial of topotecan, cisplatin and bevacizumab for recurrent or persistent cervical cancer. Gynecol Oncol. 2013;130(1):64-68. 
14. Du F, Zheng Z, Shi S, et al. S-1 and Cisplatin with or without nimotuzumab for patients with untreated unresectable or metastatic gastric cancer: a randomized, open-label phase 2 trial. Medicine (Baltimore). 2015;94(23):e958.

15. Liu ZG, Zhao Y, Tang J, et al. Nimotuzumab combined with concurrent chemoradiotherapy in locally advanced nasopharyngeal carcinoma: a retrospective analysis. Oncotarget. 2016;7(17):24429-24435.

16. Hertlein L, Lenhard M, Kirschenhofer A, et al. Cetuximab monotherapy in advanced cervical cancer: a retrospective study with five patients. Arch Gynecol Obstet. 2011;283(1):109-113.
17. Santin AD, Sill MW, McMeekin DS, et al. Phase II trial of cetuximab in the treatment of persistent or recurrent squamous or non-squamous cell carcinoma of the cervix: a Gynecologic Oncology Group study. Gynecol Oncol. 2011;122(3):495-500.

18. Jin T, Zhu Y, Luo JL, et al. Prospective phase II trial of nimotuzumab in combination with radiotherapy and concurrent capecitabine in locally advanced rectal cancer. Int J Colorectal Dis. 2015;30(3):337-345.

\section{Publish your work in this journal}

OncoTargets and Therapy is an international, peer-reviewed, open access journal focusing on the pathological basis of all cancers, potential targets for therapy and treatment protocols employed to improve the management of cancer patients. The journal also focuses on the impact of management programs and new therapeutic agents and protocols on

\section{Dovepress}

patient perspectives such as quality of life, adherence and satisfaction. The manuscript management system is completely online and includes a very quick and fair peer-review system, which is all easy to use. Visit http://www.dovepress.com/testimonials.php to read real quotes from published authors.

Submit your manuscript here: http://www.dovepress.com/oncotargets-and-therapy-journal 